ALEA, Lat. Am. J. Probab. Math. Stat. 13, 79-94 (2016)

DOI: 10.30757 /ALEA.v13-04

\title{
Persistence exponent for random processes in Brownian scenery
}

\section{Fabienne Castell, Nadine Guillotin-Plantard and Frédérique Watbled}

Aix-Marseille Université, CNRS, Centrale Marseille, Institut de Mathématiques de Marseille, UMR 7373, 13453 Marseille. France.

E-mail address: fabienne.castell@univ-amu.fr

Institut Camille Jordan, CNRS UMR 5208, Université de Lyon, Université Lyon 1, 43, Boulevard du 11 novembre 1918, 69622 Villeurbanne, France.

E-mail address: nadine.guillotin@univ-lyon1.fr

LMBA, CNRS UMR 6205, Université de Bretagne Sud, Campus de Tohannic, BP 573, 56017 Vannes, France.

E-mail address: watbled@univ-ubs.fr

Abstract. In this paper we consider the persistence properties of random processes in Brownian scenery, which are examples of non-Markovian and non-Gaussian processes. More precisely we study the asymptotic behaviour for large $T$, of the probability $\mathbb{P}\left[\sup _{t \in[0, T]} \Delta_{t} \leq 1\right]$ where $\Delta_{t}=\int_{\mathbb{R}} L_{t}(x) d W(x)$. Here $W=\{W(x) ; x \in \mathbb{R}\}$ is a two-sided standard real Brownian motion and $\left\{L_{t}(x) ; x \in \mathbb{R}, t \geq 0\right\}$ is the local time of some self-similar random process $Y$, independent from the process $W$. We thus generalize the results of Castell et al. (2013) where the increments of $Y$ were assumed to be independent.

\section{Introduction}

Let $W=\{W(x) ; x \in \mathbb{R}\}$ be a standard two-sided real Brownian motion and $Y=\{Y(t) ; t \geq 0\}$ be a real-valued self-similar process of index $\gamma \in(0,2)$ (i.e. for any $\left.c>0,\{Y(c t) ; t \geq 0\} \stackrel{(d)}{=}\left\{c^{\gamma} Y(t) ; t \geq 0\right\}\right)$, with stationary increments. When it exists, we will denote by $\left\{L_{t}(x) ; x \in \mathbb{R}, t \geq 0\right\}$ a version of the local time of the process $\{Y(t) ; t \geq 0\}$. The process $L$ satisfies the occupation density formula: for

Received by the editors January 15, 2015; accepted November 6, 2015.

2010 Mathematics Subject Classification. 60F05; 60F17; 60G15; 60G18; 60K37.

Key words and phrases. Random scenery, First passage time, One-sided barrier problem, Onesided exit problem, Survival exponent, Persistence.

This research was supported by the french ANR project MEMEMO2 2010 BLAN 0125. F. Watbled thanks the IRMAR, CNRS UMR 6625, University of Rennes 1, for its hospitality. 
each bounded measurable function $f: \mathbb{R} \rightarrow \mathbb{R}$ and for each $t \geq 0$,

$$
\int_{0}^{t} f(Y(s)) d s=\int_{\mathbb{R}} f(x) L_{t}(x) d x .
$$

The processes $W$ and $Y$ are defined on the same probability space and are assumed to be independent. We consider the random process in Brownian scenery $\left\{\Delta_{t} ; t \geq 0\right\}$ defined as

$$
\Delta_{t}=\int_{\mathbb{R}} L_{t}(x) d W(x) .
$$

The process $\Delta$ is itself a self-similar process of index $h$ with stationary increments, with

$$
h:=1-\frac{\gamma}{2}
$$

This process can be seen as a mixture of Gaussian processes, but it is neither Gaussian nor Markovian. It appeared independently in the mathematical literature (see Kesten and Spitzer (1979); Borodin (1979)), and in the physics literature (see Matheron and de Marsily (1980)) where it was originally introduced to model diffusion in layered white-noise velocity field. Note indeed that $\Delta_{t}$ can formally be written as $\int_{0}^{t} \dot{W}(Y(s)) d s$. Thus, it describes the horizontal motion of a tracer particle in a $(1+1)$-dimensional medium, where the motion of the particle along the vertical direction $y$ is described by the process $Y$, while along the horizontal direction, the particle is driven by the white noise velocity field $\dot{W}(y)$, that depends only on the vertical coordinate $y$. The process $\left\{\Delta_{t} ; t \geq 0\right\}$ provides a simple example of anomalous super-diffusion, being for instance of order $t^{3 / 4}$ when $Y$ is a Brownian motion. In this paper, we are interested in the persistence probability of the process $\Delta$, i.e. the asymptotic behaviour of

$$
\mathcal{F}(T):=\mathbb{P}\left[\sup _{t \in[0, T]} \Delta_{t} \leq 1\right]
$$

as $T \rightarrow+\infty$. The study of the one-sided exit problem of random processes is a classical issue in probability. One usually gets polynomial decay of the persistence probability: $\mathcal{F}(T) \asymp T^{-\theta}$ for a non-negative $\theta$, often called persistence exponent, or survival exponent. Classical examples, where this exponent can be computed, include random walks or Lévy processes, and we refer the reader to the recent survey papers Aurzada and Simon (2012); Bray et al. (2013) for an account of models where the persistence exponent is known. However, there are relevant physical situations where this exponent remains unknown (see for instance Majumdar (1999); Bray et al. (2013)). The persistence exponent of the Brownian motion in Brownian scenery was studied by Redner (1990, 1997), and Majumdar (2003). Based on physical arguments, numerical simulations and analogy with fractional Brownian motion, Redner and Majumdar conjectured the value of the persistence exponent. In Castell et al. (2013), their conjecture was proved up to logarithmic factors, when the process $Y$ is a stable Lévy process with index $\delta \in(1,2]$. The proof of Castell et al. (2013) depends heavily on the increments independence of the process $Y$ and the question raises if it is possible to compute the persistence exponent without it. The aim of this paper is to answer this question, and to provide assumptions on $Y$ allowing to compute the persistence exponent of the random process in Brownian scenery.

(H1): There exists a continuous version $\left\{L_{t}(x) ; x \in \mathbb{R}, t \geq 0\right\}$ of the local time of $Y$; 
(H2): $Y$ is a self-similar process of index $\gamma \in(0,2)$;

(H3): For every $T>0,\{Y(T-t)-Y(T) ; t \in[0, T]\}$ has the same distribution as either $\{Y(t) ; t \in[0, T]\}$ or $\{-Y(t) ; t \in[0, T]\}$;

(H4): Let $V_{1}:=\int L_{1}^{2}(x) d x$ be the self-intersection local time of $Y$. There exist a real number $\alpha>1$, and positive constants $C, c$ such that for any $x \geq 0$,

$$
\mathbb{P}\left[V_{1} \geq x\right] \leq C \exp \left(-c x^{\alpha}\right)
$$

(H5): There exist a real number $\beta>0$, and positive constants $C, c$ such that for any $x>0$,

$$
\mathbb{P}\left[V_{1} \leq x\right] \leq C \exp \left(-c x^{-\beta}\right) .
$$

Our main result is the following one.

Theorem 1.1. Assume (H1) to (H5) hold. There exists a constant $c>0$, such that for large enough $T$,

$$
T^{-\gamma / 2}(\ln T)^{-c} \leq \mathbb{P}\left[\sup _{t \in[0, T]} \Delta_{t} \leq 1\right] \leq T^{-\gamma / 2}(\ln T)^{+c} .
$$

Our main achievement in this paper is to remove the increments independence assumption on $\{Y(t) ; t \geq 0\}$, which was crucial in Castell et al. (2013). Another interesting issue would be to obtain the persistence exponent for $\{W(x) ; x \in \mathbb{R}\}$ being a stable Lévy process. This seems delicate, our proof relying on the fact that the process $\{\Delta(t) ; t \geq 0\}$ is a Gaussian process, conditionally to $\{Y(t) ; t \geq 0\}$.

The paper is organized as follows. Section 2 provides three examples of processes satisfying (H1) to (H5), including the stable Lévy process with index $\delta \in(1,2]$, and thus generalizing the result of Castell et al. (2013). Section 3 states some useful properties of the process $\Delta$. Section 4 is devoted to the proof of Theorem 1.1.

\section{Some examples}

2.1. A sufficient condition for (H5). In this section we assume that (H1) holds and we provide a sufficient condition on $\{Y(t) ; t \geq 0\}$ allowing to check (H5). Let us first compare $V_{1}$ with $\max _{s \in[0,1]}|Y(s)|$. By noting that

$$
1=\int_{\left\{|x| \leq \max _{s \in[0,1]}|Y(s)|\right\}} L_{1}(x) d x \leq V_{1}^{1 / 2} \sqrt{2 \max _{s \in[0,1]}|Y(s)|},
$$

we deduce that

$$
\frac{1}{2 \max _{s \in[0,1]}|Y(s)|} \leq V_{1} .
$$

Lemma 2.1. Assume that (H1) holds and that there exist positive constants $C$ and $c$, and a real number $\beta>0$ such that for all $x>0$,

$$
\mathbb{P}\left[\max _{s \in[0,1]}|Y(s)| \geq x\right] \leq C \exp \left(-c x^{\beta}\right) .
$$

Then (H5) holds true.

Proof: It is a direct consequence of (2.1) that for all $x>0$,

$$
\mathbb{P}\left[V_{1} \leq x\right] \leq \mathbb{P}\left[\max _{s \in[0,1]}|Y(s)| \geq \frac{1}{2 x}\right] \leq C \exp \left(-c 2^{-\beta} x^{-\beta}\right) .
$$


2.2. Stable Lévy processes. A process $Y=\{Y(t) ; t \geq 0\}$ is a strictly stable Lévy process with index $\delta \in(1,2]$ if it is a process with stationary and independent increments, such that $Y(0)=0$ and for any $t \geq 0$ and $u \in \mathbb{R}$,

$$
\mathbb{E}\left[e^{i u Y(t)}\right]=\exp \left\{-c|u|^{\delta} t(1+i \zeta \operatorname{sgn}(u) \tan (\pi \delta / 2))\right\},
$$

where $\zeta \in[-1,1]$, and $c$ is a positive scale parameter.

Lemma 2.2. The stable Lévy process with index $\delta \in(1,2]$ satisfies (H1) to (H5) with $\gamma=\frac{1}{\delta}, \alpha=\delta$, and $\beta=\frac{\delta}{2 \delta-1}$.

Proof: It is immediate that the Lévy process is self-similar of index $1 / \delta$ and that for every $T>0$,

$$
\{Y(T-t)-Y(T) ; t \in[0, T]\} \stackrel{(d)}{=}\{-Y(t) ; t \in[0, T]\}
$$

then (H2) and (H3) are satisfied.

A continuous version of the local time exists, since $\delta>1$ (this was proved by Boylan (1964)), so it satisfies (H1). Moreover, Corollary 5.6 in Khoshnevisan and Lewis (1998) states that there exist positive constants $C$ and $\xi$ s.t. for every $x>0$,

$$
\mathbb{P}\left[V_{1} \geq x\right] \leq C e^{-\xi x^{\delta}},
$$

which gives (H4). To prove (H5) we let $V_{t}:=\int L_{t}^{2}(x) d x$ be the self-intersection local time of $Y$ up to time $t$ for $t \geq 0$, and we show that there exists $l>0$ such that

$$
\mathbb{P}\left[V_{t} \leq 1\right] \leq e^{-l t} \text { for } t \text { large enough. }
$$

As $\left\{V_{t} ; t \geq 0\right\}$ is self-similar of index $\frac{2 \delta-1}{\delta},(2.3)$ implies that for every positive $\varepsilon$ small enough,

$$
\mathbb{P}\left[V_{1} \leq \varepsilon\right]=\mathbb{P}\left[V_{\varepsilon^{-\frac{\delta}{2 \delta-1}}} \leq 1\right] \leq e^{-l \varepsilon^{-\frac{\delta}{2 \delta-1}}},
$$

which gives (H5). To prove (2.3) we show that the function defined on $\mathbb{R}^{+}$by

$$
f(t)=\log \mathbb{P}\left[V_{t} \leq 1\right]
$$

is subadditive. Let us fix $s, t$ in $[0,+\infty)$. We consider the process $Y^{(s)}:=\left\{Y_{u}^{(s)} ; u \geq\right.$ $0\}$ defined by

$$
Y_{u}^{(s)}=Y(u+s)-Y(s)
$$

its local time $\left\{L_{u}^{(s)}(x) ; x \in \mathbb{R}, u \geq 0\right\}$, and its self-intersection local time $\left\{V_{u}^{(s)} ; u \geq\right.$ $0\}$. Note that

$$
L_{t+s}(x)=L_{s}(x)+L_{t}^{(s)}(x-Y(s)) .
$$

Hence

$$
V_{t+s} \geq V_{s}+V_{t}^{(s)}
$$

The process $Y$ being a Lévy process, $V_{t}^{(s)}$ and $V_{s}$ are independent and $V_{t}^{(s)}$ has the same law as $V_{t}$. Therefore

$$
\mathbb{P}\left[V_{t+s} \leq 1\right] \leq \mathbb{P}\left[V_{s} \leq 1 ; V_{t}^{(s)} \leq 1\right]=\mathbb{P}\left[V_{s} \leq 1\right] \mathbb{P}\left[V_{t} \leq 1\right] .
$$

Thus $f$ is subadditive, which implies that $\frac{f(t)}{t}$ converges, as $t \rightarrow+\infty$, towards a limit $-l=\inf _{t>0} \frac{f(t)}{t}$ (see Hammersley (1962)). It remains to show that $l$ is strictly 
positive. As $-l$ is less than or equal to $f(1)$, it is enough to show that $\mathbb{P}\left[V_{1}>1\right]>0$. But thanks to (2.1) and Proposition 10.3 of Fristedt (1974),

$$
\mathbb{P}\left[V_{1}>1\right] \geq \mathbb{P}\left[\max _{s \in[0,1]}|Y(s)|<\frac{1}{2}\right]>0,
$$

and this concludes the proof of (2.3).

2.3. Fractional Brownian motion. The fractional Brownian motion of Hurst index $H \in(0,1)$ is the real centered Gaussian process $\left\{B_{H}(t) ; t \geq 0\right\}$ with covariance function

$$
\mathbb{E}\left[B_{H}(t) B_{H}(s)\right]=\frac{1}{2}\left(t^{2 H}+s^{2 H}-|t-s|^{2 H}\right) .
$$

Lemma 2.3. The fractional Brownian motion with Hurst index $H \in(0,1)$ satisfies (H1) to (H5) with $\gamma=H, \alpha=1 / H, \beta=2$.

Proof: It follows readily from the definition that $B_{H}$ is self-similar with index $H$ and satisfies $(H 3)$. The existence of a jointly continuous version of its local time process for $H \in(0,1)$ is a classical fact (see for instance paragraph 22 in Geman and Horowitz (1980)). Theorem 1 of Hu et al. (2008) asserts that there exists $\lambda_{0}>0$ such that for every $0<\lambda<\lambda_{0}$,

$$
\mathbb{E}\left[e^{\lambda V_{1}^{1 / H}}\right]<\infty
$$

which implies (H4) with $\alpha=1 / H$. Finally, (H5) follows from Lemma 2.1 and Fernique's estimation (Fernique (1975), Theorem 4.1.1): there exists $c_{F}>0$ such that for any $x \geq \sqrt{5}$,

$$
\mathbb{P}\left[\max _{s \in[0,1]}\left|B_{H}(s)\right| \geq c_{F} x\right] \leq 10 \int_{x}^{+\infty} e^{-\frac{v^{2}}{2}} d v .
$$

This implies (H5) with $\beta=2$.

2.4. Iterated Brownian motion. Let $\{B(x) ; x \in \mathbb{R}\}$ be a two-sided real standard Brownian motion, and let $\{\tilde{B}(t) ; t \geq 0\}$ be a real standard Brownian motion, independent of $\{B(x) ; x \in \mathbb{R}\}$. The iterated Brownian motion is the process $\{Y(t) ; t \geq 0\}$ defined by

$$
Y(t)=B(\tilde{B}(t)) .
$$

Lemma 2.4. The iterated Brownian motion satisfies (H1) to (H5) with $\gamma=1 / 4$, and $\alpha=\beta=4 / 3$.

Proof: The self-similarity of the iterated Brownian motion is a direct consequence of the self-similarity and independence of both Brownian motions. The assertion $(H 3)$ follows once again from the independence of $B$ and $\tilde{B}$, the increments stationarity of $B$ and the fact that $\tilde{B}$ satisfies $(H 3)$.

The existence and joint continuity of the local times of iterated Brownian motion were proved in Burdzy and Khoshnevisan (1995); Csáki et al. (1996). Let us prove 
(H5). For $x>0$,

$$
\begin{aligned}
\mathbb{P}\left[\max _{s \in[0,1]}|Y(s)| \geq x\right] & \leq \mathbb{P}\left[\max _{s \in[0,1]}|\tilde{B}(s)| \geq x^{2 / 3}\right]+\mathbb{P}\left[\max _{|u| \leq x^{2 / 3}}|B(u)| \geq x\right] \\
& \leq \mathbb{P}\left[\max _{s \in[0,1]}|\tilde{B}(s)| \geq x^{2 / 3}\right]+\mathbb{P}\left[\max _{u \in\left[0, x^{2 / 3}\right]}|B(u)| \geq x\right] \\
& \leq 2 \mathbb{P}\left[\max _{s \in[0,1]}|B(s)| \geq x^{2 / 3}\right] \\
& \leq 4 \mathbb{P}\left[\max _{s \in[0,1]} B(s) \geq x^{2 / 3}\right]=4 \mathbb{P}\left[|B(1)| \geq x^{2 / 3}\right] .
\end{aligned}
$$

This proves (H5) with $\beta=4 / 3$ using Lemma 2.1.

To prove (H4), we use the uniform norm on the local times of iterated Brownian motion proved in Lemma 4 of Xiao (1998): there exists a constant $K>0$ such that for any even integer $n$,

$$
\sup _{x \in \mathbb{R}} \mathbb{E}\left[\left(L_{1}(x)\right)^{n}\right] \leq K^{n}(n !)^{3 / 4} .
$$

Since $\int_{\mathbb{R}} L_{1}(x) d x=1$, Hölder's inequality implies that for any integer $n \geq 1$,

$$
V_{1}^{n}=\left(\int_{\mathbb{R}} L_{1}(x)^{2} d x\right)^{n} \leq \int_{\mathbb{R}} L_{1}(x)^{n+1} d x=\int_{-\max _{s \in[0,1]}|Y(s)|}^{\max _{s \in[0,1]}|Y(s)|} L_{1}(x)^{n+1} d x .
$$

Therefore,

$$
\begin{aligned}
\mathbb{E}\left[V_{1}^{n}\right] & \leq \int_{\mathbb{R}} \mathbb{E}\left[L_{1}(x)^{n+1} \mathbf{1}_{|x| \leq \max _{s \in[0,1]}|Y(s)|}\right] d x \\
& \leq \int_{\mathbb{R}} \sqrt{\mathbb{E}\left[L_{1}(x)^{2(n+1)}\right]} \sqrt{\mathbb{P}\left[\max _{s \in[0,1]}|Y(s)| \geq|x|\right]} d x \\
& \leq K^{n+1}((2 n+2) !)^{3 / 8} \int_{\mathbb{R}} \sqrt{\mathbb{P}\left[\max _{s \in[0,1]}|Y(s)| \geq|x|\right]} d x,
\end{aligned}
$$

where we used (2.7) in the last inequality. By (2.6), the integral in (2.8) is finite. We deduce then from Stirling's formula that there exists a constant $C>0$ such that for any integer $n \geq 1$,

$$
\mathbb{E}\left[V_{1}^{n}\right] \leq C^{n} n^{\frac{3}{4} n} .
$$

Hence, for any $x>0$, and any integer $n \geq 1$,

$$
\mathbb{P}\left[V_{1} \geq x\right] \leq x^{-n} C^{n} n^{\frac{3}{4} n} .
$$

Optimizing over the values of $n$ leads to take $n=\left\lceil e^{-1}\left(\frac{x}{C}\right)^{4 / 3}\right\rceil$, so that $x^{-n} C^{n} n^{\frac{3}{4} n}$ $\simeq \exp \left(-\frac{3}{4 e}\left(\frac{x}{C}\right)^{4 / 3}\right.$ ) for large $x$. This proves that the iterated Brownian motion satisfies (H4) with $\alpha=4 / 3$. 


\section{Auxiliary statements on $\left(\Delta_{t} ; t \geq 0\right)$}

For a certain class of stochastic processes $\left\{X_{t} ; t \geq 0\right\}$ (to be specified below), Molchan (1999) proved that the asymptotic behavior of

$$
\mathbb{P}\left[\sup _{t \in[0, T]} X_{t} \leq 1\right]
$$

is related to the quantity

$$
I(T):=\mathbb{E}\left[\left(\int_{0}^{T} e^{X_{t}} d t\right)^{-1}\right] .
$$

We refer to Aurzada (2011) where the relationship between both quantities is clearly explained as well as the heuristics. Moreover $I(T)$ can be estimated by the following result.

Theorem 3.1 (Statement 1, Molchan (1999)). Let $\left\{X_{t} ; t \geq 0\right\}$ be a continuous process, self-similar with index $h>0$ such that for every $T>0$,

$$
\left\{X_{T-t}-X_{T} ; t \in[0, T]\right\} \stackrel{(d)}{=}\left\{X_{t} ; t \in[0, T]\right\} .
$$

Moreover assume that for every $\theta>0$,

$$
\mathbb{E}\left[\exp \left(\theta \max _{t \in[0,1]}\left|X_{t}\right|\right)\right]<+\infty
$$

Then, as $T \rightarrow+\infty$,

$$
\mathbb{E}\left[\left(\int_{0}^{T} e^{X_{t}} d t\right)^{-1}\right]=h T^{-(1-h)}\left(\mathbb{E}\left[\max _{t \in[0,1]} X_{t}\right]+o(1)\right) .
$$

Before applying this result to our random process $\Delta$ we first establish some useful facts concerning it. First we show that the process satisfies

$$
\left\{\Delta_{T-t}-\Delta_{T} ; t \in[0, T]\right\} \stackrel{(d)}{=}\left\{\Delta_{t} ; t \in[0, T]\right\}
$$

and has stationary increments (Lemma 3.2). Next we provide an exponential upper bound for the tail of $\Delta_{1}$, from which we deduce the Kolmogorov-Centsov continuity criterion (Lemma 3.3). Finally we show that the process satisfies a maximal inequality (Lemma 3.4).

Lemma 3.2. Assume $(H 1)$ and (H3). The process $\Delta$ satisfies for every $T>0$,

$$
\left\{\Delta_{T-t}-\Delta_{T} ; t \in[0, T]\right\} \stackrel{(d)}{=}\left\{\Delta_{t} ; t \in[0, T]\right\} .
$$

Therefore, it has stationary increments, i.e. for every $s>0,\left\{\Delta_{t+s}-\Delta_{s} ; t \geq 0\right\} \stackrel{(d)}{=}$ $\left\{\Delta_{t} ; t \geq 0\right\}$.

Proof: Conditionally to $\{Y(t) ; t \geq 0\}$, the process $\left\{\Delta_{T-t}-\Delta_{T} ; t \in[0, T]\right\}$ is a centered Gaussian process with (random) covariance function:

$$
C_{T}(s, t)=\int_{\mathbb{R}}\left(L_{T}(x)-L_{T-t}(x)\right)\left(L_{T}(x)-L_{T-s}(x)\right) d x .
$$

Note that $L_{T}(x)-L_{T-t}(x)=L_{t}^{(T)}(x-Y(T))$, where $\left\{L_{t}^{(T)}(x) ; t \in[0, T], x \in\right.$ $\mathbb{R}\}$ is the local time process of $Y_{t}^{(T)}:=Y(T-t)-Y(T)$. Hence, $C_{T}(s, t)=$ 
$\int_{\mathbb{R}} L_{t}^{(T)}(x) L_{s}^{(T)}(x) d x$. Now, if $Y^{(T)}$ has the same distribution as $Y$ (respectively $-Y)$, then $\left\{L_{t}^{(T)}(x) ; t \in[0, T], x \in \mathbb{R}\right\}$ has the same law as $\left\{L_{t}(x) ; t \in[0, T], x \in \mathbb{R}\right\}$ (respectively $\left\{L_{t}(-x) ; t \in[0, T], x \in \mathbb{R}\right\}$ ). We deduce then that in both cases, $\left\{C_{T}(s, t) ; s, t \in[0, T]\right\}$ is distributed as the conditional covariance of $\left\{\Delta_{t} ; t \in[0, T]\right\}$ with respect to $Y$. Hence,

$$
\left\{\Delta_{T-t}-\Delta_{T} ; t \in[0, T]\right\} \stackrel{(d)}{=}\left\{\Delta_{t} ; t \in[0, T]\right\} .
$$

Concerning the increments stationarity, fix $s>0$, and $0<t_{1}<\cdots<t_{n}$. Let $T>t_{n}+s$ be fixed.

$$
\begin{aligned}
\left(\Delta_{t_{1}+s}-\right. & \left.\Delta_{s}, \cdots, \Delta_{t_{n}+s}-\Delta_{s}\right) \\
& \stackrel{(d)}{=}\left(\Delta_{T-t_{1}-s}-\Delta_{T-s}, \cdots, \Delta_{T-t_{n}-s}-\Delta_{T-s}\right) \text { by time reversal at time } T, \\
& \stackrel{(d)}{=}\left(\Delta_{t_{1}}, \cdots, \Delta_{t_{n}}\right) \text { by time reversal at time } T-s .
\end{aligned}
$$

Lemma 3.3. Assume (H1) to (H4) hold. There exist $C>0$ and $\delta>0$ such that for any $x>0$,

$$
\mathbb{P}\left[\Delta_{1} \geq x\right] \leq C \exp \left(-\delta x^{\frac{2 \alpha}{1+\alpha}}\right) .
$$

Hence, for every $a \geq 1$,

$$
\left(\mathbb{E}\left|\Delta_{t}-\Delta_{s}\right|^{a}\right)^{1 / a}=C(a)|t-s|^{h}, t, s \geq 0
$$

where $C(a) \leq c a^{\nu}$ with $\nu:=\frac{1}{2}\left(1+\frac{1}{\alpha}\right)$. In particular the process satisfies the Kolmogorov-Centsov criterion of continuity.

Proof: Conditionally to the process $Y$, the random variable $\Delta_{1}$ is a real centered Gaussian variable with variance $V_{1}$. For each $u \in \mathbb{R}$, let

$$
\Phi(u)=\frac{1}{\sqrt{2 \pi}} \int_{u}^{+\infty} e^{-\frac{s^{2}}{2}} d s .
$$

Then for $x>0$ and $\theta>0$,

$$
\begin{aligned}
\mathbb{P}\left[\Delta_{1} \geq x\right]=\int_{0}^{+\infty} \Phi\left(x z^{-1 / 2}\right) \mathbb{P}_{V_{1}}(d z)= & \int_{0}^{x^{\theta}} \Phi\left(x z^{-1 / 2}\right) \mathbb{P}_{V_{1}}(d z) \\
& +\int_{x^{\theta}}^{+\infty} \Phi\left(x z^{-1 / 2}\right) \mathbb{P}_{V_{1}}(d z) \\
\leq & \Phi\left(x^{1-\frac{\theta}{2}}\right)+\mathbb{P}\left[V_{1} \geq x^{\theta}\right],
\end{aligned}
$$

where we used that the function $z \mapsto \Phi\left(x z^{-1 / 2}\right)$ is non decreasing with values in $[0,1]$. Using (H4) and the classical inequality

$$
\Phi(u) \leq \frac{1}{\sqrt{2 \pi} u} e^{-u^{2} / 2} \text { for every } u>0,
$$

and choosing $\theta=\frac{2}{1+\alpha}$, we obtain that for $x$ large enough,

$$
\mathbb{P}\left[\Delta_{1} \geq x\right] \leq \frac{x^{-\alpha /(1+\alpha)}}{\sqrt{2 \pi}} e^{-\frac{1}{2} x^{2 \alpha /(1+\alpha)}}+C e^{-c x^{2 \alpha /(1+\alpha)}} \leq C e^{-\delta x^{2 \alpha /(1+\alpha)}},
$$

with $\delta=\min (c, 1 / 2)$. This implies (3.2) for every $x>0$. 
Let us prove (3.3). The increments of the process $\Delta$ being stationary (see Lemma (3.2)), by self-similarity, we have for every $t, s \geq 0$,

$$
\mathbb{E}\left[\left|\Delta_{t}-\Delta_{s}\right|^{a}\right]=|t-s|^{h a} \mathbb{E}\left[\left|\Delta_{1}\right|^{a}\right] .
$$

Now, using (3.2) and the symmetry of $\Delta_{1}$,

$$
\begin{aligned}
\mathbb{E}\left[\left|\Delta_{1}\right|^{a}\right] & =\int_{0}^{+\infty} \mathbb{P}\left[\left|\Delta_{1}\right| \geq x^{1 / a}\right] d x \leq 2 \int_{0}^{+\infty} \mathbb{P}\left[\Delta_{1} \geq x^{1 / a}\right] d x \\
& \leq C \int_{0}^{+\infty} \exp \left(-\delta x^{\frac{2 \alpha}{a(1+\alpha)}}\right) d x=C a \delta^{-\frac{a(1+\alpha)}{2 \alpha}} \Gamma\left(\frac{a(1+\alpha)}{2 \alpha}\right) .
\end{aligned}
$$

Hence, it follows from Stirling's formula that $C(a)=\mathbb{E}\left[\left|\Delta_{1}\right|^{a}\right]^{1 / a} \leq C a^{(1+\alpha) /(2 \alpha)}$.

Lemma 3.4. Assume (H1) and (H4) hold. Let T, $x \geq 0$. Then

$$
\begin{gathered}
\mathbb{P}\left[\max _{s \in[0, T]} \Delta_{s} \geq x \mid Y\right] \leq 2 \mathbb{P}\left[\Delta_{T} \geq x \mid Y\right], \\
\mathbb{P}\left[\max _{s \in[0, T]} \Delta_{s} \geq x\right] \leq 2 \mathbb{P}\left[\Delta_{T} \geq x\right] .
\end{gathered}
$$

Proof: Conditionally to the process $Y$, the process $\left\{\Delta_{t} ; t \geq 0\right\}$ is a centered Gaussian process on $\mathbb{R}$ with covariance function

$$
\mathbb{E}\left[\Delta_{s} \Delta_{t} \mid Y\right]=\int_{\mathbb{R}} L_{s}(x) L_{t}(x) d x .
$$

Moreover for any $t \geq s \geq 0$,

$$
\begin{aligned}
\mathbb{E}\left[\Delta_{t}^{2} \mid Y\right]-\mathbb{E}\left[\Delta_{s}^{2} \mid Y\right]-\mathbb{E}\left[\left(\Delta_{t}-\Delta_{s}\right)^{2} \mid Y\right] & =\int_{\mathbb{R}}\left(L_{t}^{2}(x)-L_{s}^{2}(x)\right. \\
& \left.-\left(L_{t}(x)-L_{s}(x)\right)^{2}\right) d x \\
& =\int_{\mathbb{R}} 2 L_{s}(x)\left(L_{t}(x)-L_{s}(x)\right) d x \geq 0,
\end{aligned}
$$

hence applying Proposition 2.2 in Khoshnevisan and Lewis (1998), we deduce the inequality (3.5). By integrating we obtain the maximal inequality (3.6).

We are now in position to use Theorem 3.1 and to state the main result of this section.

Proposition 3.5. Assume (H1) to (H4) hold. As $T \rightarrow+\infty$,

$$
\mathbb{E}\left[\left(\int_{0}^{T} e^{\Delta_{t}} d t\right)^{-1}\right]=\left(1-\frac{\gamma}{2}\right) T^{-\gamma / 2}\left(\mathbb{E}\left[\max _{t \in[0,1]} \Delta_{t}\right]+o(1)\right) .
$$

Proof: It follows easily from assumption (H2), that the process $\left\{\Delta_{t} ; t \geq 0\right\}$ is selfsimilar with index $h:=1-\frac{\gamma}{2}$. From Lemma 3.2, it satisfies (3.1). From (3.3) and Kolmogorov-Centsov continuity criterion, we can assume that it is continuous. Hence, to apply Theorem 3.1, it is enough to prove that for every $\theta>0$,

$$
\mathbb{E}\left[\exp \left(\theta \max _{t \in[0,1]}\left|\Delta_{t}\right|\right)\right]<+\infty
$$


Let $\theta>0$. We have

$$
\begin{aligned}
\mathbb{E}\left[\exp \left(\theta \max _{t \in[0,1]}\left|\Delta_{t}\right|\right)\right] & =\int_{0}^{\infty} \mathbb{P}\left[\exp \left(\theta \max _{t \in[0,1]}\left|\Delta_{t}\right|\right) \geq \lambda\right] d \lambda \\
& \leq 2+\int_{2}^{\infty} \mathbb{P}\left[\max _{t \in[0,1]}\left|\Delta_{t}\right| \geq \frac{\ln (\lambda)}{\theta}\right] d \lambda .
\end{aligned}
$$

Since the process $\left\{\Delta_{t} ; t \geq 0\right\}$ is symmetric and satisfies the maximal inequality (3.6) of Lemma 3.4,

$$
\begin{aligned}
\mathbb{P}\left[\max _{t \in[0,1]}\left|\Delta_{t}\right| \geq \frac{\ln (\lambda)}{\theta}\right] & \leq 2 \mathbb{P}\left[\max _{t \in[0,1]} \Delta_{t} \geq \frac{\ln (\lambda)}{\theta}\right] \\
& \leq 4 \mathbb{P}\left[\Delta_{1} \geq(\ln \lambda) / \theta\right] .
\end{aligned}
$$

We apply the inequality (3.2) of Lemma 3.3, and since the function

$$
\lambda \rightarrow \exp \left(-\delta((\ln \lambda) / \theta)^{2 \alpha /(1+\alpha)}\right)
$$

is integrable at infinity for any $\alpha>1$, the assertion (3.7) follows.

\section{Proof of Theorem 1.1}

In this section, we prove upper and lower bounds on the persistence probability $\mathbb{P}\left[\sup _{t \in[0, T)} \Delta(t) \leq 1\right]$.

In the case of fractional Brownian motion $\left\{B_{H}(t) ; t \geq 0\right\}$, Aurzada's proof of the lower bound (see Aurzada (2011)), rests on both following arguments: the fractional Brownian motion satisfies the hypothesis of Theorem 3.1, and it satisfies the equality (valid for $a$ large enough)

$$
\left(\mathbb{E}\left|B_{H}(t)-B_{H}(s)\right|^{a}\right)^{1 / a}=C(a)|t-s|^{H}, t, s \geq 0
$$

with $C(a) \leq c a^{\nu}$, for some $c$ and $\nu>0$. We showed (see Lemma 3.3 and Proposition $3.5)$ that these conditions are also satisfied by our random process $\Delta$. Therefore the proof of Aurzada (2011) allows us to derive the lower bound in Theorem 1.1.

Let us give a sketch of the proof. Let $\eta \in\left(\frac{h}{2}, h\right)$ and let us fix $a$ such that $a>\frac{2}{h}$ and $\eta<h-\frac{1}{a}$. From Lemma 2.1 in Scheutzow (2009) and equation (3.3) we deduce that for any $0<\varepsilon \leq 1$, for every $s, t \in[0,1]$ such that $|t-s| \leq \varepsilon$,

$$
\left|\Delta_{t}-\Delta_{s}\right| \leq S \varepsilon^{\eta}
$$

where $S$ is a random variable such that

$$
\mathbb{E}\left[S^{a}\right] \leq \frac{\left(d a^{\nu}\right)^{a}}{2^{(a h-1)-a \eta}-1},
$$

with $d$ a constant depending only on $c$ and $h$. The quantity $I(T)$ defined in Section 3 can now be related to the Laplace transform $g(T):=\mathbb{E}\left[e^{-T^{h} \Delta_{1}^{*}}\right]$, where $\Delta_{1}^{*}=$ $\sup _{t \in[0,1]} \Delta_{t}$. Indeed, using self similarity, $\int_{0}^{T} e^{\Delta_{t}} d t \stackrel{(d)}{=} T \int_{0}^{1} e^{T^{h} \Delta_{t}} d t$. For any $\epsilon \in(0,1]$, let $\mathcal{V}$ be a $\varepsilon$-neighborhood of the maximum of $\left\{\Delta_{t}, t \in[0,1]\right\}$. By (4.2),

$$
\int_{0}^{1} e^{T^{h} \Delta_{t}} d t \geq e^{T^{h} \Delta_{1}^{*}} \int_{\mathcal{V}} e^{T^{h}\left(\Delta_{t}-\Delta_{1}^{*}\right)} d t \geq e^{T^{h} \Delta_{1}^{*}} e^{-T^{h} S \varepsilon^{\eta}} \varepsilon .
$$


Choosing $\varepsilon:=\left(T^{h} S\right)^{-1 / \eta} \wedge 1$, we obtain

$$
\int_{0}^{1} e^{T^{h} \Delta_{t}} d t \geq e^{T^{h} \Delta_{1}^{*}} e^{-1} \frac{1}{\left(T^{h} S\right)^{1 / \eta}+1}
$$

Hence,

$$
I(T) \leq e\left(\mathbb{E}\left[T^{h / \eta} S^{1 / \eta} e^{-T^{h} \Delta_{1}^{*}}\right]+g(T)\right) T^{-1}
$$

Using Hölder's inequality with $p:=a \eta>1$ and the moment of order $a$ of the random variable $S$, we get

$$
e^{-1} I(T) \leq \frac{d^{1 / \eta} a^{\nu / \eta}}{\left(2^{a(h-\eta)-1}-1\right)^{1 /(a \eta)}} g(T)^{1-1 /(a \eta)} T^{-1+h / \eta}+T^{-1} g(T) .
$$

Fix $\delta \in(0,1)$, and set $a=\log T(\log \log T)^{-\delta}, \eta=h-2 / a$. Note that $h-\eta=$ $2 / a>1 / a$ and $a \eta=a h-2>1$ for $T$ large enough. From (4.3),

$$
\left(k I(T) a^{-\nu / \eta} T^{-2 /(a \eta)}\right)^{1 /(1-1 /(a \eta))} \leq g(T),
$$

for some constant $k$. From Proposition 3.5, we know that $I(T) \sim c T^{-(1-h)}$ for $T$ large. Then,

$$
g(T) \geq T^{-(1-h)}(\log T)^{-\nu / h+o(1)}
$$

The lower bound is then obtained from direct computations. Indeed, by selfsimilarity,

$$
\begin{aligned}
\mathbb{P}\left[\sup _{t \in[0, T]} \Delta_{t} \leq 1\right] & =\mathbb{P}\left[\Delta_{1}^{*} \leq T^{-h}\right] \\
& \geq \mathbb{E}\left[e^{-T^{h} \log (T)^{2} \Delta_{1}^{*}}\right]-e^{-\log (T)^{2}} \\
& \geq T^{-(1-h)} \log (T)^{-(2(1-h)+\nu) / h+o(1)}-e^{-\log (T)^{2}} .
\end{aligned}
$$

As in Molchan (1999) and Aurzada (2011), the main idea in the proof of the upper bound in (1.2), is to bound $I(T)$ from below by restricting the expectation to a well-chosen set of paths.

Conditionally to $Y$, the process $\left\{\Delta_{t} ; t \geq 0\right\}$ is a centered Gaussian process such that for every $0 \leq t<s$,

$$
\begin{gathered}
\mathbb{E}\left[\Delta_{t} \Delta_{s} \mid Y\right]=\int_{\mathbb{R}} L_{t}(x) L_{s}(x) d x \geq 0, \\
\mathbb{E}\left[\Delta_{t}\left(\Delta_{s}-\Delta_{t}\right) \mid Y\right]=\int_{\mathbb{R}} L_{t}(x)\left(L_{s}(x)-L_{t}(x)\right) d x \geq 0,
\end{gathered}
$$

since $t \rightarrow L_{t}(x)$ is a.s. increasing for all $x \in \mathbb{R}$. It follows then from Slepian's lemma (see the proof of Theorem 3 in Slepian (1962) or Lemma 1.2.5 in Baumgarten (2013)), that for every $0 \leq u<v<w$ and every real numbers $a, b$,

$$
\mathbb{P}\left[\sup _{t \in[u, v]} \Delta_{t} \leq a, \sup _{t \in[v, w]} \Delta_{t} \leq b \mid Y\right] \geq \mathbb{P}\left[\sup _{t \in[u, v]} \Delta_{t} \leq a \mid Y\right] \mathbb{P}\left[\sup _{t \in[v, w]} \Delta_{t} \leq b \mid Y\right]
$$




$$
\begin{aligned}
\mathbb{P}\left[\sup _{t \in[u, v]} \Delta_{t} \leq a, \sup _{t \in[v, w]}\left(\Delta_{t}-\Delta_{v}\right) \leq b \mid Y\right] \\
\geq \mathbb{P}\left[\sup _{t \in[u, v]} \Delta_{t} \leq a \mid Y\right] \mathbb{P}\left[\sup _{t \in[v, w]}\left(\Delta_{t}-\Delta_{v}\right) \leq b \mid Y\right]
\end{aligned}
$$

Let $a_{T}=(\ln T)^{a}$, where $a>0$ will be chosen later; let $\alpha_{T}:=\frac{\ln T}{1+\ln T}$, which belongs to $(0,1)$ and set $\beta_{T}=\sqrt{V_{a_{T}}}$ where $V_{a_{T}}:=\int_{\mathbb{R}} L_{a_{T}}^{2}(x) d x$. Let us define the random function

$$
\phi(t):= \begin{cases}1 & \text { for } 0 \leq t<a_{T}, \\ 1-\beta_{T} & \text { for } a_{T} \leq t \leq T,\end{cases}
$$

which is $Y$-measurable. Clearly, we have

$$
\mathbb{E}\left[\left(\int_{0}^{T} e^{\Delta_{t}} d t\right)^{-\alpha_{T}} \mid Y\right] \geq\left(\int_{0}^{T} e^{\phi(t)} d t\right)^{-\alpha_{T}} \mathbb{P}\left[\forall t \in[0, T], \Delta_{t} \leq \phi(t) \mid Y\right]
$$

By Slepian's lemma (see (4.4)), we have

$$
\begin{aligned}
\mathbb{P}\left[\forall t \in[0, T], \Delta_{t}\right. & \leq \phi(t) \mid Y] \\
& \geq \mathbb{P}\left[\forall t \in\left[0, a_{T}\right], \Delta_{t} \leq 1 \mid Y\right] \mathbb{P}\left[\forall t \in\left[a_{T}, T\right], \Delta_{t} \leq 1-\beta_{T} \mid Y\right] .
\end{aligned}
$$

Remark that

$$
\begin{aligned}
& \mathbb{P}\left[\forall t \in\left[a_{T}, T\right], \Delta_{t} \leq 1-\beta_{T} \mid Y\right] \\
\geq & \mathbb{P}\left[\Delta_{a_{T}} \leq-\beta_{T} ; \forall t \in\left[a_{T}, T\right], \Delta_{t}-\Delta_{a_{T}} \leq 1 \mid Y\right] \\
\geq & \mathbb{P}\left[\Delta_{a_{T}} \leq-\beta_{T} \mid Y\right] \mathbb{P}\left[\forall t \in\left[a_{T}, T\right], \Delta_{t}-\Delta_{a_{T}} \leq 1 \mid Y\right],
\end{aligned}
$$

by Slepian's lemma (see (4.5)). Note that

$$
\mathbb{P}\left[\Delta_{a_{T}} \leq-\beta_{T} \mid Y\right]=\Phi\left(\beta_{T} V_{a_{T}}^{-1 / 2}\right)=\Phi(1) .
$$

Moreover, it is easy to check that when $T$ goes to infinity,

$$
\int_{0}^{T} e^{\phi(t)} d t=O\left(a_{T}+T e^{-\beta_{T}}\right) .
$$

In the following $C$ is a constant whose value may change but does not depend on $T$. Then we can write that for $T$ large enough

$$
\begin{aligned}
& \mathbb{E}\left[\left(\int_{0}^{T} e^{\Delta_{t}} d t\right)^{-\alpha_{T}} \mid Y\right] \\
& \geq C\left(a_{T}+T e^{-\beta_{T}}\right)^{-\alpha_{T}} \mathbb{P}\left[\sup _{t \in\left[0, a_{T}\right]} \Delta_{t} \leq 1 \mid Y\right] \mathbb{P}\left[\sup _{t \in\left[a_{T}, T\right]}\left(\Delta_{t}-\Delta_{a_{T}}\right) \leq 1 \mid Y\right] .
\end{aligned}
$$

Next we use the maximal inequality (3.5) of Lemma 3.4 to write

$$
\begin{aligned}
\mathbb{P}\left[\sup _{t \in\left[0, a_{T}\right]} \Delta_{t} \leq 1 \mid Y\right] & =1-\mathbb{P}\left[\sup _{t \in\left[0, a_{T}\right]} \Delta_{t} \geq 1 \mid Y\right] \geq 1-2 \mathbb{P}\left[\Delta_{a_{T}} \geq 1 \mid Y\right] \\
& =\mathbb{P}\left[|Z| \leq V_{a_{T}}^{-1 / 2} \mid Y\right]
\end{aligned}
$$


where $Z$ is a Gaussian variable $\mathcal{N}(0,1)$ independent of $Y$, from which we deduce that there exists a constant $c>0$ such that

$$
\mathbb{P}\left[\sup _{t \in\left[0, a_{T}\right]} \Delta_{t} \leq 1 \mid Y\right] \geq c \min \left(V_{a_{T}}^{-1 / 2}, 1\right) .
$$

Injecting (4.7) into (4.6) we get that for $T$ large enough,

$$
\begin{aligned}
\mathbb{P}\left[\operatorname { s u p } _ { t \in [ a _ { T } , T ] } \left(\Delta_{t}-\right.\right. & \left.\left.\Delta_{a_{T}}\right) \leq 1 \mid Y\right] \\
\leq C \mathbb{E} & {\left[\left(\int_{0}^{T} e^{\Delta_{t}} d t\right)^{-\alpha_{T}} \mid Y\right]\left(a_{T}+T e^{-V_{a_{T}}^{1 / 2}}\right)^{\alpha_{T}} \max \left(V_{a_{T}}^{1 / 2}, 1\right) . }
\end{aligned}
$$

By integrating and using successively Hölder's inequality with $p_{T}=\frac{1}{\alpha_{T}}, \frac{1}{q_{T}}+$ $\frac{1}{p_{T}}=1$, Jensen's inequality, the inequality $(x+y)^{\alpha_{T}} \leq x^{\alpha_{T}}+y^{\alpha_{T}}$ for $x, y>0$, and Proposition 3.5, we get that for $T$ large enough,

$$
\begin{aligned}
& \mathbb{P}\left[\sup _{t \in\left[a_{T}, T\right]}\left(\Delta_{t}-\Delta_{a_{T}}\right) \leq 1\right] \\
& \leq C \mathbb{E}\left[\left(\int_{0}^{T} e^{\Delta_{t}} d t\right)^{-1}\right]^{1 / p_{T}} \\
& \times \mathbb{E}\left[\left(a_{T}^{\alpha_{T}} \max \left(V_{a_{T}}^{1 / 2}, 1\right)+T^{\alpha_{T}} e^{\left.\left.-\alpha_{T} V_{a_{T}}^{1 / 2} \max \left(V_{a_{T}}^{1 / 2}, 1\right)\right)^{q_{T}}\right]^{1 / q_{T}}}\right.\right. \\
& \leq C T^{-\frac{\gamma}{2 p_{T}}}\left\|f_{1}+f_{2}\right\|_{q_{T}} \leq C T^{-\frac{\gamma}{2 p_{T}}}\left(\left\|f_{1}\right\|_{q_{T}}+\left\|f_{2}\right\|_{q_{T}}\right),
\end{aligned}
$$

with

$$
f_{1}=a_{T}^{\alpha_{T}} \max \left(V_{a_{T}}^{1 / 2}, 1\right), \quad f_{2}=T^{\alpha_{T}} e^{-\alpha_{T} V_{a_{T}}^{1 / 2}} \max \left(V_{a_{T}}^{1 / 2}, 1\right) .
$$

The lefthand term is greater than the quantity we want to bound from above, since by stationarity,

$$
\mathbb{P}\left[\sup _{t \in\left[a_{T}, T\right]}\left(\Delta_{t}-\Delta_{a_{T}}\right) \leq 1\right]=\mathbb{P}\left[\sup _{t \in\left[0, T-a_{T}\right]} \Delta_{t} \leq 1\right] \geq \mathbb{P}\left[\sup _{t \in[0, T]} \Delta_{t} \leq 1\right] .
$$

Concerning the righthand term, we recall that $\alpha_{T}=\frac{1}{p_{T}}=\frac{\ln T}{1+\ln T}$ and $\frac{1}{q_{T}}=1-\alpha_{T}=$ $\frac{1}{1+\ln T}$. Hence, when $T$ goes to infinity, $T^{-\frac{\gamma}{2 p_{T}}} \leq C T^{-\frac{\gamma}{2}}$. Therefore,

$$
\mathbb{P}\left[\sup _{t \in[0, T]} \Delta_{t} \leq 1\right] \leq C T^{-\frac{\gamma}{2}}\left(\left\|f_{1}\right\|_{q_{T}}+\left\|f_{2}\right\|_{q_{T}}\right) .
$$

It remains to prove that $\left\|f_{1}\right\|_{q_{T}}$ and $\left\|f_{2}\right\|_{q_{T}}$ are bounded by logarithmic terms.

$$
\left\|f_{1}\right\|_{q_{T}}=a_{T}^{\alpha_{T}} \mathbb{E}\left[V_{a_{T}}^{q_{T} / 2} \mathbf{1}_{V_{a_{T}} \geq 1}+\mathbf{1}_{V_{a_{T}} \leq 1}\right]^{1 / q_{T}} \leq a_{T}^{\alpha_{T}}\left(\mathbb{E}\left[V_{a_{T}}^{q_{T}}\right]^{1 /\left(2 q_{T}\right)}+1\right) .
$$

By $(\mathrm{H} 2), V_{a_{T}} \stackrel{\mathcal{L}}{=} a_{T}^{2-\gamma} V_{1}$. Therefore,

$$
\mathbb{E}\left[V_{a_{T}}^{q_{T}}\right]^{1 /\left(2 q_{T}\right)}=a_{T}^{1-\frac{\gamma}{2}} \mathbb{E}\left[V_{1}^{q_{T}}\right]^{1 /\left(2 q_{T}\right)} .
$$

As in (3.4), it follows from (H4) that

$$
\mathbb{E}\left[V_{1}^{m}\right] \leq C c^{-m / \alpha} \frac{m}{\alpha} \Gamma\left(\frac{m}{\alpha}\right) \text { for every } m \in \mathbb{N},
$$


so that using Stirling's formula, it is easy to show that for $T$ large enough

$$
\mathbb{E}\left[V_{1}^{q_{T}}\right]^{1 / 2 q_{T}} \leq C(\ln T)^{\frac{1}{2 \alpha}} .
$$

We conclude that for $T$ large enough

$$
\left\|f_{1}\right\|_{q_{T}} \leq C(\ln T)^{a\left(2-\frac{\gamma}{2}\right)+\frac{1}{2 \alpha}} .
$$

Let us now turn our attention to $\left\|f_{2}\right\|_{q_{T}}$.

$$
\begin{aligned}
\left\|f_{2}\right\|_{q_{T}} & \leq T^{\alpha_{T}} \mathbb{E}\left[e^{-2 q_{T} \alpha_{T} V_{a_{T}}^{1 / 2}}\right]^{1 / 2 q_{T}} \mathbb{E}\left[\max \left(V_{a_{T}}, 1\right)^{q_{T}}\right]^{1 / 2 q_{T}} \\
& \leq C T(\ln T)^{a\left(1-\frac{\gamma}{2}\right)+\frac{1}{2 \alpha}} \mathbb{E}\left[e^{-2 q_{T} \alpha_{T} a_{T}^{1-\frac{\gamma}{2}} \sqrt{V_{1}}}\right]^{1 / 2 q_{T}} .
\end{aligned}
$$

Let us note $\lambda_{T}=2 q_{T} \alpha_{T} a_{T}^{1-\frac{\gamma}{2}}=2(\ln T)^{1+a\left(1-\frac{\gamma}{2}\right)}$. Then using (H5),

$$
\begin{aligned}
\mathbb{E}\left[e^{-\lambda_{T} V_{1}^{1 / 2}}\right] & \leq \int_{0}^{+\infty} \mathbb{P}\left[V_{1} \leq \frac{u^{2}}{\lambda_{T}^{2}}\right] e^{-u} d u \\
& \leq C \int_{0}^{+\infty} e^{-c \frac{\lambda_{T}^{2 \beta}}{u^{2 \beta}}} e^{-u} d u
\end{aligned}
$$

for some constants $c$ and $C$. We perform the change of variable $u=\lambda_{T}^{\frac{2 \beta}{1+2 \beta}} v$ in the preceding integral. This yields

$$
\begin{aligned}
\mathbb{E}\left[e^{-\lambda_{T} V_{1}^{1 / 2}}\right] & \leq C \lambda_{T}^{2 \beta /(1+2 \beta)} \int_{0}^{+\infty} e^{-\lambda_{T}^{\frac{2 \beta}{1+2 \beta}}\left(v+c v^{-2 \beta}\right)} d v \\
& \leq C \lambda_{T}^{2 \beta /(1+2 \beta)} \int_{1}^{+\infty} e^{-\lambda_{T}^{\frac{2 \beta}{1+2 \beta}} v} d v+C \lambda_{T}^{2 \beta /(1+2 \beta)} \int_{0}^{1} e^{-\lambda_{T}^{\frac{2 \beta}{1+2 \beta}} c v^{-2 \beta}} d v \\
& \leq C e^{-\lambda_{T}^{\frac{2 \beta}{1+2 \beta}}}+C e^{-c \lambda_{T}^{\frac{2 \beta}{1+2 \beta}}} \\
& \leq C e^{-c \lambda_{T}^{\frac{2 \beta}{1+2 \beta}}},
\end{aligned}
$$

for some other constants $c$ and $C$. This leads to

$$
\left\|f_{2}\right\|_{q_{T}} \leq C T(\ln T)^{a\left(1-\frac{\gamma}{2}\right)+\frac{1}{2 \alpha}} e^{-c(\ln T)^{\frac{2 \beta}{1+2 \beta}\left(1+a\left(1-\frac{\gamma}{2}\right)\right)-1}} .
$$

We choose $a$ such that $a\left(1-\frac{\gamma}{2}\right)>1+\frac{1}{\beta}$. Then $T e^{-c(\ln T)^{\frac{2 \beta}{1+2 \beta}\left(1+a\left(1-\frac{\gamma}{2}\right)\right)-1}}$ remains bounded, and when $T$ goes to infinity, we get

$$
\left\|f_{2}\right\|_{q_{T}} \leq C(\ln T)^{c}
$$

for some constant $c>1+\frac{1}{\beta}+\frac{1}{2 \alpha}$. From (4.8), (4.9) and (4.10) we deduce that for $T$ large enough,

$$
\mathbb{P}\left[\sup _{t \in[0, T]} \Delta_{t} \leq 1\right] \leq C T^{-\frac{\gamma}{2}}(\ln T)^{c},
$$

with $c>\frac{\beta+1}{\beta} \frac{4-\gamma}{2-\gamma}+\frac{1}{2 \alpha}$.

Acknowledgments. We are grateful to Yueyun Hu for stimulating discussions. 


\section{References}

F. Aurzada. On the one-sided exit problem for fractional Brownian motion. Electron. Commun. Probab. 16, 392-404 (2011). MR2831079.

F. Aurzada and T. Simon. Persistence probabilities \& exponents. ArXiv Mathematics e-prints 16 (2012). arXiv: 1203.6554.

C. Baumgarten. Persistence of sums of independent random variables, iterated processes and fractional Brownian motion. Ph.D. thesis, Technische Universität Berlin, Fakultät II - Mathematik und Naturwissenschaften (2013). DOI: 10.14279/depositonce-3620.

A.N. Borodin. A limit theorem for sums of independent random variables defined on a recurrent random walk. Dokl. Akad. Nauk SSSR 246 (4), 786-787 (1979). MR543530.

E.S. Boylan. Local times for a class of Markoff processes. Illinois J. Math. 8, 19-39 (1964). MR0158434.

A. Bray, S. Majumdar and G. Schehr. Persistence and first-passage properties in non-equilibrium systems. Advances in Physics 62 (3), 225-361 (2013). DOI: 10.1080/00018732.2013.803819.

K. Burdzy and D. Khoshnevisan. The level sets of iterated Brownian motion. In Séminaire de Probabilités, XXIX, volume 1613 of Lecture Notes in Math., pages 231-236. Springer, Berlin (1995). MR1459464.

F. Castell, N. Guillotin-Plantard, F. Pène and B. Schapira. On the one-sided exit problem for stable processes in random scenery. Electron. Commun. Probab. 18, no. 33, 7 (2013). MR3064992.

E. Csáki, M. Csörgö, A. Földes and P. Révész. The local time of iterated Brownian motion. J. Theoret. Probab. 9 (3), 717-743 (1996). MR1400596.

$\mathrm{X}$. Fernique. Régularité des trajectoires des fonctions aléatoires gaussiennes. In École d'Été de Probabilités de Saint-Flour, IV-1974, pages 1-96. Lecture Notes in Math., Vol. 480. Springer, Berlin (1975). MR0413238.

B. Fristedt. Sample functions of stochastic processes with stationary, independent increments. In Advances in probability and related topics, Vol. 3, pages 241-396. Dekker, New York (1974). MR0400406.

D. Geman and J. Horowitz. Occupation densities. Ann. Probab. 8 (1), 1-67 (1980). MR556414.

J.M. Hammersley. Generalization of the fundamental theorem on sub-additive functions. Proc. Cambridge Philos. Soc. 58, 235-238 (1962). MR0137800.

Y. Hu, D. Nualart and J. Song. Integral representation of renormalized selfintersection local times. J. Funct. Anal. 255 (9), 2507-2532 (2008). MR2473265.

H. Kesten and F. Spitzer. A limit theorem related to a new class of self-similar processes. Z. Wahrsch. Verw. Gebiete 50 (1), 5-25 (1979). MR550121.

D. Khoshnevisan and T.M. Lewis. A law of the iterated logarithm for stable processes in random scenery. Stochastic Process. Appl. 74 (1), 89-121 (1998). MR1624017.

S. Majumdar. Persistence in nonequilibrium systems. Current Science $\mathbf{7 7}$ (3), 370-375 (1999).

S. Majumdar. Persistence of a particle in the Matheron-de Marsily velocity field. Phys. Rev. E 68, 050101 (2003). DOI: 10.1103/PhysRevE.68.050101.

G. Matheron and G. de Marsily. Is transport in porous media always diffusive? a counterexample. Water Resources Research 16 (5), 901-917 (1980). DOI: 
10.1029/WR016i005p00901.

G. M. Molchan. Maximum of a fractional Brownian motion: probabilities of small values. Comm. Math. Phys. 205 (1), 97-111 (1999). MR1706900.

S. Redner. Superdiffusion in random velocity fields. Physica A: Statistical Mechanics and its Applications 168 (1), 551-560 (1990). DOI: 10.1016/ 03784371(90)90408-K.

S. Redner. Survival probability in a random velocity field. Phys. Rev. E 56, 49674972 (1997). DOI: 10.1103/PhysRevE.56.4967.

M. Scheutzow. Chaining techniques and their application to stochastic flows. In Trends in stochastic analysis, volume 353 of London Math. Soc. Lecture Note Ser., pages 35-63. Cambridge Univ. Press, Cambridge (2009). MR2562150.

D. Slepian. The one-sided barrier problem for Gaussian noise. Bell System Tech. J. 41, 463-501 (1962). MR0133183.

Y. Xiao. Local times and related properties of multidimensional iterated Brownian motion. J. Theoret. Probab. 11 (2), 383-408 (1998). MR1622577. 